\title{
CONDIÇÕES DE TRABALHO E SAÚDE DE DOCENTES DE PÓS-GRADUAÇÃO STRICTO SENSU DE LETRAS E LINGUÍSTICA
}

\author{
WORKING AND HEALTH CONDITIONS IN EDUCATORS OF STRICTO \\ SENSU POSTGRADUATE PROGRAMS IN LETTERS AND LINGUISTICS
}

\author{
Maynara Fernanda Carvalho Barreto \\ Universidade Estadual de Londrina, Londrina, Paraná, Brasil \\ maynarabarreto@hotmail.com
}

Maria José Quina Galdino Universidade Estadual do Norte do Paraná, Bandeirantes, Paraná, Brasil mariagaldino@uenp.edu.br

Rosemeri Passos Baltazar Machado Universidade Estadual de Londrina, Londrina, Paraná, Brasil rosemeri@sercomtel.com.br

Frederico Augusto Garcia Fernandes Universidade Estadual de Londrina, Londrina, Paraná, Brasil fredericofernandes3@gmail.com

Maria do Carmo Fernandez Lourenço Haddad Universidade Estadual de Londrina, Londrina, Paraná, Brasil carmohaddad@gmail.com

\begin{abstract}
Resumo: A pesquisa objetiva investigar as condições de trabalho e saúde de docentes de pósgraduação stricto sensu de Letras e Linguística do Brasil. Trata-se de um estudo transversal desenvolvido com docentes vinculados a cursos de 155 Programas de Pós-Graduação de Letras e Linguística do Brasil, reconhecidos ou recomendados pela Coordenação de Aperfeiçoamento de Pessoal de Nível Superior (CAPES). Fizeram parte do estudo 654 docentes, com participação proporcional de cada região do Brasil. Os dados foram analisados por estatística descritiva. Os resultados demonstraram que, quanto às condições de trabalho, $80 \%$ referiram estarem satisfeitos com o trabalho na pós-graduação, apesar de considerarem que o ritmo e a intensidade do trabalho docente interferem negativamente em suas vidas $(65,7 \%)$. Em relação às condições de saúde, verificamos que $52,2 \%$ dos docentes apresentam-se satisfeitos com a sua saúde e afirmaram ter uma boa qualidade de vida $(61,2 \%)$. Em contrapartida, afirmaram não estarem satisfeitos com o sono $(56,6 \%)$ e se sentirem exaustos emocionalmente (31,9\%). Esses resultados demonstram uma dualidade entre as condições de trabalho e a saúde, apresentando uma demanda em relação ao desenvolvimento de estratégias individuais e organizacionais para o aprimoramento do trabalho docente.
\end{abstract}

Palavras-chave: Docentes; Condições de Trabalho; Nível de Saúde; Programas de Pós-Graduação; 
Letras e Linguística

Abstract: This study aims to investigate the working and health conditions in educators of stricto sensu postgraduate programs in Letters and Linguistics in Brazil. This study is a transversal study performed with educators of 155 programs recommended by Coordenação de Aperfeiçoamento de Pessoal de Nível Superior of Brazil. In this study, 654 educators accepted to answer the questions about their working and health conditions, with a proportional participation of each region of Brazil. Data were analyzed by descriptive statistics. The results about working conditions showed that $80 \%$ of educators reported being satisfied with their work in postgraduate program, although they consider that the pace and intensity of teaching work negatively interferes with their lives $(65,7 \%)$. About the health conditions, $52,2 \%$ educators were satisfied with their health and with a good quality of life $(61,2 \%)$. However, the participants reported not being satisfied with sleep $(56.6 \%)$ and feeling emotionally exhausted (31.9\%). These results shows a duality between working and health conditions, presenting a demand regarding the development of individual and organizational strategies for the improvement of teaching work.

Keywords: Faculty; Workinng Conditions; Health Status; Graduate Programs; Letters and Linguistics

\section{Introdução}

O trabalho docente, caracterizado pelo processo de ensino e aprendizagem, apresenta-se como essencial na promoção da educação, qualificação dos saberes e desenvolvimento de gerações. A atividade profissional e o processo de trabalho do docente implicam em formar profissionais que possam transformar os conhecimentos aprendidos, no ambiente em que estão inseridos, e fortalecer o processo de trabalho dos professores durante a sua formação (LEITE; NOGUEIRA, 2017; SCHEUCH; HAUFE; SEIBT, 2015).

No que se refere aos docentes universitários e atividades desempenhadas, destacamse as relacionadas ao ensino, pesquisa e extensão demarcadas pela produção de textos, livros, orientações, participação em eventos com apresentação de trabalhos, organização de eventos, emissão de pareceres para as agências de fomento e revistas, participação em bancas de defesa e de concurso, as disciplinas ministradas na graduação e na pós-graduação e as atividades de orientação (Iniciação Científica, Mestrado e Doutorado). No entanto, professores vinculados a programas de pós-graduação apresentam um intenso ritmo de produção acadêmica, sobretudo em relação à publicação de artigos científicos, sob o risco de serem pouco valorizados ou até mesmo excluídos do quadro de docentes permanentes dos programas (SOUZA et al., 2017).

Além disso, o processo de trabalho do docente de pós-graduação e as suas condições de trabalho podem resultar em insatisfações do professor e condições de saúde inadequadas (SOUZA et al., 2017). Nesse sentido, surgem os problemas de saúde mental como as doenças psicossomáticas, sinais e sintomas como exaustão, fadiga, dor de cabeça, estresse ocasionado por fatores físicos, químicos ou ergonômicos (SCHEUCH; HAUFE; SEIBT, 2015), sociais, psicológicos, biológicos e do meio ambiente (BRASIL, 1999).

As condições de trabalho e a saúde docente constituem temas relevantes, uma vez que tem sido evidenciado o crescimento do adoecimento docente como resultado das condições de administração do trabalho e promoção de saúde insatisfatórias (CORTEZ et al., 2017). Além disso, a organização do trabalho produz efeitos sobre a saúde mental dos trabalhadores e a identificação dos fatores preveníveis é fundamental para que sejam trabalhados no processo laboral (GIONGO; MONTEIRO; SOBROSA, 2015).

Aliada à importância de conhecer mais profundamente o fenômeno da saúde e sua relação com o trabalho docente, acrescenta-se a não identificação de estudos sobre as condições de trabalho e saúde de docentes de Programas de Pós-Graduação em Letras e Linguística (PPGLL) do Brasil. Dessa forma, esse estudo teve por objetivo investigar as 
condições de trabalho e saúde de docentes de pós-graduação stricto sensu de Letras e Linguística do Brasil.

\section{Método}

Trata-se de um estudo descritivo, do tipo transversal, com abordagem quantitativa, realizado com docentes de 95 universidades brasileiras, sendo 19 de instituições de ensino superior privadas e 76 públicas. A população de estudo foi constituída por 3.008 docentes vinculados aos 155 PPGLL, reconhecidos pela Coordenação de Aperfeiçoamento de Pessoal de Nível Superior (CAPES), conforme dados disponíveis na Plataforma Sucupira.

Em relação aos critérios de elegibilidade, foram convidados a participar do estudo os docentes vinculados a, pelo menos, um dos PPGLL, desde que seus e-mails estivessem disponíveis nos sítios eletrônicos institucionais de cada Programa, dos quais 2.753 docentes foram convidados a participar da pesquisa e $654(23,8 \%)$ aceitaram o convite, com participação proporcional de cada região do Brasil.

Para a obtenção dos dados, foi desenvolvida uma plataforma eletrônica de coleta de dados por meio de um sítio na internet, denominado Mubble, para abrigar um questionário semiestruturado contendo variáveis sociodemográficas e ocupacionais, especialmente elementos característicos do processo de trabalho docente. Para o desenvolvimento da plataforma, foram utilizadas ferramentas tecnológicas como as linguagens de programação JavaScript para o desenvolvimento das interfaces do sistema, e Hypertext Preprocessor (PHP) para a construção das operações relacionadas ao servidor do sistema, em conjunto com o sistema de gerenciamento de dados $M y S Q L$.

A partir do desenvolvimento da plataforma, ela foi configurada para enviar, via email, o questionário de coleta de dados junto ao convite para participar da pesquisa. A coleta de dados foi realizada entre fevereiro e agosto de 2019 e, posteriomente, foi elaborado um banco de dados a fim de realizar as análises descritivas, com base em dados referentes à frequência e às porcentagens.

$\mathrm{O}$ desenvolvimento do estudo atendeu às normas de ética em pesquisas nacionais e internacionais, incluindo aprovação do Comitê de Ética em Pesquisa da Universidade Estadual de Londrina (UEL), conforme Parecer $\mathrm{n}^{\circ}$. 2.347.839, CAAE: 79006017.0.0000.5231. Todos os participantes foram consultados quanto ao aceite em participar do estudo por meio do Termo de Consentimento Livre e Esclarecido (TCLE).

\section{Resultados}

Dos 654 docentes que aceitaram participar da pesquisa, 414 (63,3\%) eram mulheres, $443(67,7 \%)$ casados, $74(11,3 \%)$ separados, $117(17,9 \%)$ solteiros e 20 (3\%) viúvos. Em relação à faixa etária, 122 (18\%) possuíam entre 29 a 40 anos, 249 (38\%) entre 41 a 50 anos, $177(27 \%)$ entre 51 a 59 anos e $106(16 \%)$ acima de 60 anos.

Em relação à titulação dos participantes da pesquisa, destaca-se mais da metade $(\mathrm{n}=374 ; 57,2 \%)$ com pós-doutorado. Quanto à atuação nos PPGLL, $515(78,7 \%)$ atuavam em um PPGLL, $129(19,7 \%)$ em dois e $10(1,6 \%)$ docentes em três programas.

Sobre o vínculo empregatício, $569(87 \%)$ estavam vinculados à instituição de forma efetiva, $63(9,63 \%)$ celetistas e $22(3,37 \%)$ como visitantes. Quanto aos Programas e Universidades de atuação, 369 (56,4\%) pertenciam a instituições federais, $214(32,7 \%)$ a estaduais e $71(10,9 \%)$ a instituições privadas. Em relação à localização geográfica dos Cursos, a região Sudeste $(\mathrm{n}=229 ; 35 \%)$ foi a mais representativa, seguida da região Sul $(n=177 ; 27,1 \%)$, Nordeste $(n=140 ; 21,4 \%)$, Norte $(n=36 ; 5,5 \%)$ e Centro Oeste $(n=72 ; 11 \%)$. Dos PPGLL, mais da metade dos programas $(n=362 ; 55,4 \%)$ apresentam pontuação cinco a sete no conceito Capes. 
Em relação à satisfação com o trabalho docente e condições de trabalho, os resultados indicaram que a maioria $(\mathrm{n}=523 ; 80 \%)$ referiram estar satisfeitos com o trabalho na pós-graduação. Em contrapartida, consideraram que o ritmo e a intensidade do trabalho docente exercem influências negativas em suas vidas ( $n=430 ; 65,7 \%)$.

Sobre os sentimentos e pensamentos dos docentes de deixarem os PPGLL, 275 (42\%) afirmaram nunca terem pensado sobre essa possibilidade. Entretanto, $272(41,6 \%)$ pensam com frequência e $107(16,4 \%)$ referiram apresentar este sentimento às vezes. Ademais, $124(18,9 \%)$ docentes referiram sentimentos de frustração com o trabalho.

No que concerne às condições de saúde e à qualidade de vida, a maioria dos docentes apresenta satisfação em relação a sua saúde $(n=348 ; 53,2 \%)$ e com boa qualidade de vida $(\mathrm{n}=403 ; 61,2 \%)$. No entanto, $370(56,6 \%)$ dos docentes não estão satisfeitos com o sono e muitos deles $(n=307 ; 46,9 \%)$ já precisaram fazer uso de medicamentos em decorrência do processo de trabalho.

No que diz respeito à exaustão emocional, 209 (31,9\%) dos docentes reconheceram sentir-se emocionalmente exaustos. Em relação à prática de atividade física, grande parte respondeu que pratica pelo menos uma vez por semana $(n=481 ; 73,5 \%)$. No entanto, pontuaram terem poucas ou nenhuma oportunidade de atividades de lazer $(n=508 ; 77,7 \%)$.

\section{Discussão}

Os PPGLL, no Brasil, desempenham um papel fundamental na produção e consolidação do conhecimento na área enquanto ciência e tecnologia (BRASIL, 2016). As características sociodemográficas dos docentes participantes deste estudo são semelhantes ao perfil apresentado em pesquisa sobre as condições de saúde de docentes de pós-graduação (CASSANDRE, 2011). Em relação à predominância do sexo feminino, destaca-se o empoderamento da mulher na carreira docente em busca de autonomia, sobretudo no gerenciamento de sua carreira (CEZAR; SCHERER; CORSO, 2017).

No que diz respeito à titulação dos participantes da pesquisa e vínculo com os PPGLL, acrescenta-se que o desenvolvimento da carreira docente é marcado por um longo percurso que somam anos de dedicação para o aprimoramento do conhecimento e qualificação profissional (ROWE; BASTOS; PINHO, 2011). Diante disso, o perfil apresentado pelos participantes corrobora com os critérios de avaliação da CAPES e características recomendadas para o corpo docente.

A CAPES recomenda e avalia o corpo docente dos PPGLL segundo estágio pósdoutoral, experiências e produção na área de atuação, projeção nacional e internacional, participação em comissões editoriais, de agências de fomento, em comissões científicas de eventos da área e se os docentes recebem bolsas de agência de fomento (BRASIL, 2016). Todavia, embora todos os docentes apresentem o Doutorado como formação máxima, as instituições de ensino público oferecem mais oportunidades de aprimoramento profissional (ROWE; BASTOS; PINHO, 2011).

Em relação à nota dos programas, tem-se a consolidação e liderança nacional dos PPGLL como resultado da capacidade de nucleação de grupos e centros de pesquisa (BRASIL, 2017), mostrando os Programas representados nesta pesquisa como centros formadores do conhecimento alinhados aos critérios e diretrizes da CAPES.

Entre as atividades docentes estão as relacionadas às aulas ministradas nas disciplinas dos Programas, o desenvolvimento de pesquisas e captação de recursos nas agências de fomento, lideranças de grupo de pesquisa, orientação de doutorandos e/ou mestrandos, a participação de estudantes de outros níveis de formação em trabalhos de conclusão de curso e iniciação científica; elaboração de artigos científicos, emissão de 
pareceres a periódicos científicos, atuação em atividades administrativas e de ensino na graduação, entre outras atividades (BRASIL, 2016).

Como podemos observar, para a realização das atividades do docente, são necessárias muitas horas de dedicação com intenso esforço mental, físico e emocional, caracterizado, muitas vezes, por uma carga horária excessiva e alterações no comportamento do trabalhador relacionado a uma compulsão por trabalho para conciliar e obter êxito em suas atividades laborais (NIE; SUN, 2016). Essa compulsão por trabalho é definida como workaholism e caracteriza-se pela dependência psicológica de um indivíduo ao seu trabalho (ZHAO et al., 2015). Tal condição pode prejudicar as relações interpessoais dos trabalhadores e influenciar as suas condições de saúde (AGOSTINI et al., 2014; CLAUDE; HADJISTAVROPOULOS; FRIESEN, 2014; HE et al., 2014).

Neste contexto, surgem os transtornos mentais como principais responsáveis pelo afastamento do trabalho por longos períodos de tempo e, até mesmo, a necessidade do uso de medicamentos. O surgimento das patologias mentais entre os docentes afeta diretamente o ambiente de trabalho e pode interferir nos objetivos pedagógicos, uma vez que os sinais e sintomas são manifestados em forma de alienação, desumanização, apatia, insônia, gastrite, cefaleia, hipertensão arterial e uso de drogas, levando ao aumento de absenteísmo e ao sentimento de querer deixar a carreira profissional (SANTOS et al., 2016; ANDRADE; CARDOSO, 2012).

O estresse, enquanto uma das causas da exaustão emocional, é descrito por meio das reações físicas, mentais e emocionais resultantes das próprias necessidades laborais. No caso do trabalho docente, o ambiente laboral é caracterizado pela complexidade de atividades, tensão emocional, atribuições estratégicas e pedagógicas, cansaço físico e mental que podem interferir negativamente no ambiente educacional (SANTOS et al., 2016).

Em relação aos resultados apresentados neste estudo sobre as condições de saúde dos docentes e qualidade de vida, é preciso compreender trabalho e saúde como uma relação possível de apresentar sofrimento. No entanto, conforme os resultados mostraram, a maneira como cada trabalhador reage e se comporta revela o potencial de utilizar recursos internos e externos capazes de transformar o sofrimento em prazer e realização, resultando em satisfação no trabalho ou, até mesmo, uma identidade profissional alinhada à sua atuação (GIONGO; MONTEIRO; SOBROSA, 2015).

Ademais, a identidade, caracterizada pelo comprometimento afetivo com a carreira, o apego emocional, o planejamento de carreira e resiliência são fatores que influenciam positivamente o esforço instrucional. No entanto, é importante destacar que estes fatores são diferenciados em docentes de carreira pública e privada. No caso da resiliência, por exemplo, enquanto comprometimento com a carreira, os investimentos na carreira e custos emocionais podem ser maiores em docentes do ensino privado em relação ao público, justificado pelo fator segurança e proteção ao trabalho que pode ser menor nas instituições de ensino privado ou, até mesmo, o vínculo horista para grande parte dos docentes (ROWE; BASTOS; PINHO, 2011).

Sobre a qualidade de vida, embora os docentes tenham relatado estarem satisfeitos, cabe salientar que situações que elevam o nível de estresse causam distúrbios à saúde e podem resultar no comprometimento da qualidade de vida e da produtividade dos docentes (LIMA JÚNIOR; SILVA, 2014).

Em relação à exaustão emocional, insatisfação com o sono e o uso de medicamentos, esses resultados podem estar associados à divisão de trabalho docente e às responsabilidades e à carga horária laboral. A rigidez no trabalho geralmente é proporcional ao estado da saúde mental atual dos trabalhadores, o que pode explicar a percepção dos respondentes acerca de sua saúde (GIONGO; MONTEIRO; SOBROSA, 2015). 
Sobre a insatisfação com o sono dos participantes da pesquisa, destacam-se o trabalho docente e a demanda psicológica para as atividades acadêmicas sujeitos a condições de trabalho que geram sofrimento, tensão emocional e acarretam desajustes físicos como a insônia (ANDRADE; CARDOSO, 2012).

O docente e a sua relação com a visão emocional e social apresentam-se em um conflito entre suportar as condições precárias de trabalho e avançar no conhecimento. No entanto, ao mesmo tempo em que relata a desvalorização profissional e desrespeito, possui prazer com o conhecimento tecido coletivamente em seu processo de trabalho e nos resultados obtidos. Geralmente, o prazer está relacionado às atividades de ensino, pesquisa, extensão e gestão, decorrentes do vigor no enfrentamento cotidiano oportunizado pela liberdade e autonomia que possui (SOUZA; OLIVEIRA, 2013).

Em relação às condições de trabalho e sentimentos autorreferidos pelos docentes de satisfação, a subjetividade do indivíduo e as suas experiências individuais, relacionadas com o sentimento de felicidade e satisfação, podem estar relacionadas à minimização do sofrimento no processo de trabalho (HE et al., 2014; SOUZA; OLIVEIRA, 2013). Além disso, pontuamse as relações sócio-profissionais positivas, a autonomia profissional para desenvolver as atividades laborais, a infraestrutura e recursos físicos adequados ao processo de trabalho e as relações interpessoais como influenciadores na subjetividade dos trabalhadores (GIONGO; MONTEIRO; SOBROSA, 2015).

O sentimento de deixar as atividades de pós-graduação, as dificuldades na permanência no contexto das universidades e a política de sucateamento da educação são elementos presentes e, sem dúvida, significativos, pois há um forte enfrentamento de uma sobrecarga de trabalho, de pressão por produtividade, de comprometimento com o processo de formação do estudante e de constantes incorporações de atividades (SOUZA; OLIVEIRA, 2013).

Estudo sobre a satisfação no trabalho docente e motivação para deixar a profissão docente mostrou aspectos como consonância de valor, apoio de supervisão, relações com colegas, pressão de tempo e problemas de disciplina como possíveis aspectos que interferem na tomada de decisão do docente sobre a sua permanência no trabalho, independentemente das diferenças em seu sistema educacional. Além disso, apresentou essas variáveis como preditivas de exaustão emocional e relacionadas ao sentimento de pertencimento docente (SKAALVIK; SKAALVIK, 2011).

Em relação ao sentimento de pertencimento do docente, há indicativos de que a luta constante na resistência às políticas reguladoras acadêmicas e à sustentabilidade das políticas institucionais são fatores que necessitam de redes de articulação entres os programas existentes, a fim de contribuir com a formação de profissionais e, consequentemente, com a valorização do trabalho docente (VASCONCELLOS; SORDI, 2016).

Em resposta aos problemas identificados, destacam-se como possíveis estratégias de promoção à saúde e qualidade de vida de docentes, desenvolver as esferas pessoais, sociais e institucionais, por meio de uma atuação conjunta que atenda às necessidades contemporâneas em saúde (LEITE; NOGUEIRA, 2017). Como um exemplo de estratégia institucional está a realização de avaliações individuais e de aconselhamento para os docentes e a importância das profissões de ensino possuírem planos de ações organizacionais, serviços de atenção à saúde ocupacional, qualificados a partir de uma rede de profissionais da saúde como enfermeiros, psicólogos, psiquiatras e médicos especialistas (SCHEUCH; HAUFE; SEIBT, 2015).

Estudo sobre comportamentos de professores indicou a realização de intervenções preventivas como uma estratégia positiva nas crenças e percepções dos professores acerca de seu desempenho profissional e sentimento de pertencimento e valorização (DOMITROVICH et al., 2016). Nesse sentido, no que concerne às responsabilidades dos trabalhadores e, neste caso dos docentes, é preciso compreender as relações de poder, as hierarquias, o comando, o 
grau de autonomia nas atividades e as possibilidades de cooperação e comunicação como fatores possíveis de serem analisados na relação trabalho/saúde (GIONGO; MONTEIRO; SOBROSA, 2015).

Sobre a necessidade de enfrentamento do trabalhador ao adoecimento, destacam-se as estratégias e intervenções relacionadas aos fatores de prazer, sofrimento, estratégias defensivas e de mobilização subjetiva. Além disso, as necessidades dos trabalhadores identificarem qual é o sentido que encontram no trabalho com as suas atividades laborais, o motivo de estarem satisfeitos com as suas atividades docentes, a possibilidade de ajudar as pessoas e a influência que o trabalho possui nos seus hábitos de vida são fatores importantes para minimizar as complicações individuais e organizacionais (GIONGO; MONTEIRO; SOBROSA, 2015).

Em relação às estratégias de nível organizacional, é importante salientar que os fatores externos como o processo de expansão mercantil e organismos financeiros internacionais possuem influência nas mudanças no trabalho docente. Isto significa que tanto professores das universidades públicas como privadas estão alinhados à reconfiguração laboral que responda às exigências de trabalho flexíveis e ao mercado neoliberal. Assim, na área docente, tem-se a exigência por maior produtividade docente, o produtivismo acadêmico e as crescentes responsabilidades e necessidades geradas aos docentes exigem, obviamente, maior envolvimento desses profissionais. No entanto, paralelamente, têm-se os fenômenos de intensificação e precarização do trabalho com o surgimento de uma demanda de intervenções capazes de garantir melhores condições laborais (VASCONCELLOS; SORDI, 2016).

\section{Considerações Finais}

Os resultados apresentados mostram as condições de trabalho e saúde dos docentes que atuam nos PPGLL e a dualidade entre os sentimentos de prazer e sofrimento no trabalho. Diante dos resultados apresentados, os autores deste estudo propõem estratégias que podem ser implantadas e realizadas tanto em nível individual quanto organizacional para melhorar as condições de trabalho e saúde dos docentes.

Em relação às estratégias individuais, é proposto $\mathrm{o}$ autoconhecimento $\mathrm{e}$ automonitoramento das habilidades sociais, a realização de atividades físicas e mentais como pilates, meditação, terapias alternativas, apoio psicológico, identificar e reconhecer as dificuldades enfrentadas no trabalho docente e buscar estratégias para minimizá-las, bem como estabelecer acordos de convivência entre docentes e orientandos, tais como horário de estudo, participação em atividades científicas e pedagógicas comum a todos, prazos de cumprimento das atividades dos orientandos, entre outras.

Sobre as estratégias organizacionais, é proposto que as instituições ofereçam melhores condições laborais, busquem medidas de flexibilizar os horários de trabalho docente, reconheçam e divulguem o bom desempenho do trabalho docente, criem estratégias de apoio psicopedagógico aos docentes que possuem dificuldades com seus orientandos, utilizem os recursos de mídia com o objetivo de enviar informações sintéticas sobre a saúde física e mental do docente e promovam, semestralmente, momentos de reflexão com o objetivo do automonitoramento das habilidades sociais e oportunizem momentos e/ou atividades de lazer no contexto do trabalho.

\section{Referências}

Agostini, F.; et al. "Parental anxiety and stress before pediatric anesthesia: A pilot study on the effectiveness of preoperative clown intervention". Journal of Health Psychology, [s.1], v. 19 , n. 5, 2014, p. 587-601. 
Andrade, P. S.; Cardoso, T. A. "O. Prazer e dor na docência: revisão bibliográfica sobra a Síndrome de Burnout". Saúde e Sociedade, [s.1], v. 21, n. 1, 2012, p. 129-40.

BRASIL. COORDENAÇÃO DE APERFEIÇOAMENTO DE PESSOAL DE NÍVEL SUPERIOR. Documento de área 2016: Letras e Linguística. Brasília: CAPES, 2016. Disponível em: http://capes.gov.br/images/documentos/Documentos de area 2017/41 LETR_docarea_2016.pdf . Acesso em: 10 mai 2019.

BRASIL. COORDENAÇÃO DE APERFEIÇOAMENTO DE PESSOAL DE NÍVEL SUPERIOR. Relatório da Avaliação Quadrienal 2017: Linguística e Literatura. Brasília: CAPES, 2017. Disponível em: http://capes.gov.br/images/documentos/ Relatorios quadrienal 2017/20122017-letras relatoriodeavalia $\% \mathrm{C} 3 \% \mathrm{~A} 7 \% \mathrm{C} 3 \%$

A3o quadrienal2017 final.pdf. Acesso em: 14 mai 2019.

BRASIL. Ministério da Saúde. Política nacional de saúde ambiental para o setor saúde. Brasília: Secretaria de Políticas de Saúde, 1999.

Cassandre, M. P. “A saúde de docentes de pós-graduação em universidades públicas: os danos causados pelas imposições do processo avaliativo". Revista Mal Estar e Subjetividade, Fortaleza, v. 11, n. 2, 2011.

Cezar, B. G. S.; Scherer, L. A.; Corso, K. B. Empoderamento feminino na carreira de mulheres docentes: estudo em uma Universidade Federal do interior do Rio Grande do Sul. Revista de Carreiras e Pessoas, [s.1], v. 7, n. 1, 2017, p. 440-55.

Claude, J. A. J.; Hadjistavropoulos, H. D.; Friesen, L. "Exploration of health anxiety among individuals with diabetes: Prevalence and implications". Journal of Health Psychology, [s.1], v. 19, n. 2,2014 , p. 312-22.

Cortez, P. A. et al. "A saúde docente no trabalho: apontamentos a partir da literatura recente". Cadernos de Saúde Coletiva, Rio de Janeiro, v. 25, n. 1, 2017, p. 113-22.

Diehl, L.; Marin, A. H. "Adoecimento mental em professors brasileiros: revisão sistemática da literature". Estudos Interdisciplinares em Psicologia, Londrina, v. 7, n. 2, 2016, p. 64-85.

Domitrovich, C. E. et al. "How Do School-Based Prevention Programs Impact Teachers? Findings from a Randomized Trial of an Integrated Classroom Management and SocialEmotional Program”. Prevention Science, [s.1], v. 17, n. 3, 2016, p. 325-37.

Giongo, C. R.; Monteiro, J. K.; Sobrosa, G. M. R. "Psicodinâmica do trabalho no Brasil: revisão sistemática da literatura". Temas em Psicologia, Ribeirão Preto, v. 23, n. 4, 2015.

He, F.; Guan, H.; Kong, Y.; Cao, R.; Peng, J. "Some individual differences influencing the propensity to happiness: Insights from behavioral economics". Social Indicators Research, [s.1], v. 119, 2014, p. 897-908.

Leite, A. F.; Nogueira, J. A. D. "Fatores condicionantes de saúde relacionados ao trabalho de professores universitários da área da saúde: uma revisão integrativa". Revista Brasileira de Saúde Ocupacional, São Paulo, v. 42, n. 6, 2017, p. 1-15. 
Lima Júnior, J. P.; Silva, T. F. A. "Analysis of muculoskeletal disorders symptoms in professors of the University of Pernambuco - Petrolina Campus". Revista Dor, São Paulo, v. 15 , n. 4, 2014, p. 276-80.

Malik, N. A.; Bjorkqvist, K. "Occupational stress and mental and musculoskeletal health among university teachers". Eurasian Journal of Medical Investigation, Istambul, v. 2, n. 3, 2018, p. 139-147.

Nie, Y.; Sun, H. "Why do workaholics experience depression? A study with Chinese University teachers". Journal of Health Psychology, [s.1], v. 21, n. 10, 2016, p. 2339-46.

Rowe, D. E. O.; Bastos, A. V. B.; Moreno, A. P. "Comprometimento e entrincheiramento na carreira: um estudo de suas influências no esforço instrucional do docente do ensino superior”. Revista de Administração Contemporânea, [s.1], v. 15, n. 6, 2011, p. 973-92.

Santos, N. P. S. et al. "Docência universitária e o estresse: estressores nos cursos de enfermagem e medicina". Revista de Enfermagem da UFSM, Santa Maria, v. 6, n. 1, 2016.

Scheuch, K.; Haufe, E.; Seibt, R. "Teacher's Health”. Deutsches Ärzteblatt International, Berlin, v. 112, 2015, p. 347-56.

Skaalvik, E. M.; Skaalvik, S. "Teacher job satisfaction and motivation to leave the teaching profession: relations with school context, feeling of belonging, and emotional exhaustion". Teaching and Teacher Education, [s.1], v. 27, n. 6, 2011, p. 1029-1038.

Souto, B. L. C. et al. "O trabalho docente em pós-graduação: prazer e sofrimento". Revista de Enfermagem da UFSM, Santa Maria, v. 7, n. 1, 2017, p. 29-39.

Souza, A. S. S. et al. "Fatores associados à qualidade de vida no trabalho entre professores do ensino superior". Arquivos de Ciência da Saúde, São José do Rio Preto, v. 22, n. 4, 2015, p. 46-51.

Souza, K. R. et al. "A nova organização do trabalho na universidade pública: consequências coletivas da precarização na saúde dos docentes". Ciências \& Saúde Coletiva, Rio de Janeiro, v. 22, n. 11, 2017, p. 3667-76.

Souza, T. M. C.; Oliveira, C. A. H. L. "Trabalho docente: representações sociais em professores de uma universidade pública”. Psico, Porto Alegre, PUCRS, v. 44, n. 4, 2013, p. 590-600.

Vasconcellos, M. M M.; Sordi, M. R. L. "Formar professores universitários: tarefa (im)possível?". Interface, Botucatu, v. 20, n. 57, 2016, p. 403-14.

zhao, X. et al. "Dispositional optimism, self-framing and medical decision-making". International Journal of Psychology, [s.1], v. 50, n. 2, 2015, p. 121-7.

Recebido em: 20 de julho de 2019 Aceito em: 25 de outubro de 2019 Publicado em: Dezembro de 2019 